Felix Roxenlund, Robert Kruse, Hanna Östling and Maria Lodefalk*

\title{
Differential microRNA expression in placentas of small-for-gestational age neonates with and without exposure to poor maternal gestational weight gain
}

https://doi.org/10.1515/jpm-2020-0597

Received August 17, 2020; accepted February 8, 2021; published online February 23, 2021

Keywords: gestational weight gain; microRNA expression; next generation sequencing; placenta; small-for-gestational age.

To the Editor,

The primary factor determining foetal growth is nutritional supply, which is dependent on an adequate nutritional state and energy intake in the pregnant woman and a wellfunctioning placenta resulting in normal materno-foetal exchange [1]. Maternal gestational weight gain (GWG) has been shown to positively correlate with the birth weight of neonates [2], but the molecular mechanisms behind this association are not completely understood. We hypothesized that differential expression of microRNAs (miRNAs) in placenta is involved in the process linking maternal dietary intake and GWG to foetal growth [3].

miRNAs are small, non-coding RNA molecules that silence mRNAs and play an integral role in physiological and pathological processes. They have been found to associate with reductions in body weight and dietary intake among other environmental factors, and their

*Corresponding author: Maria Lodefalk, MD, PhD, Department of Paediatrics, Faculty of Medicine and Health, Örebro University, Örebro, Sweden; and Department of Paediatrics, Örebro University Hospital, 70185 Örebro, Sweden,

E-mail: maria.lodefalk@regionorebrolan.se

Felix Roxenlund, School of Medical Science, Faculty of Medicine and Health, Örebro University, Örebro, Sweden

Robert Kruse, Department of Clinical Research Laboratory, Faculty of Medicine and Health, Örebro University, Örebro, Sweden; and Inflammatory Response and Infection Susceptibility Centre (iRiSC), Faculty of Medicine and Health, Örebro University, Örebro, Sweden Hanna Östling, Department of Women's Health, Faculty of Medicine and Health, Örebro University, Örebro, Sweden expression in placenta has been reported to be altered in preeclampsia and to associate with birth of small-forgestational age (SGA) neonates [4, 5], but no previous study has reported associations with maternal GWG, as far as we know. To investigate this, we studied the expression of miRNAs in placentas from SGA neonates exposed or not to low maternal GWG (LGWG) using data from a previous study that described differences between SGA neonates and neonates with a normal birth size, all with a normal maternal GWG (NGWG) [5].

A convenience sample of 13 SGA neonates (defined as birth weight $<-2$ standard deviations according to data from a Swedish reference population [6]) exposed and nine SGA neonates not exposed to LGWG (defined as $\leq 10 \mathrm{~kg}$ ) were included in this study. The placental samples were retrieved from a sample collection at Örebro University Hospital, Örebro, Sweden. They were delivered 2007-2012 at the Department of Women's Health at the same hospital and fulfilled the following inclusion criteria: vaginal delivery in gestational week $37+0-41+6$, healthy woman with a height $>150 \mathrm{~cm}$, aged 18-42 years, and body mass index 18.5-24.9 in early pregnancy, singleton neonate without asphyxia (defined as Apgar score $\geq 7$ at $5 \mathrm{~min}$ ), chromosomal abnormality and anatomical malformation. Women smoking during pregnancy, having gestational hypertensive disease, gestational diabetes, or erythrocyte immunization were excluded, as well as deliveries induced by prostaglandins. NGWG was defined as $11.5-16.0 \mathrm{~kg}$ as recommended [2]. To secure a significant difference in GWG between the groups, each woman in the LGWG group had to have a weight gain that was $\geq 4 \mathrm{~kg}$ lower than that of a corresponding woman in the NGWG group, as described previously [5]. Written informed consent was obtained from all women. The project was approved by the Regional Board of Ethics, Uppsala, Sweden (2010/189).

Global miRNA expression in the placental samples was analysed by Next Generation Sequencing (NGS) using Illumina's technology at GATC Biotech AG in Konstanz, 
Germany, followed by technical validation of five miRNAs of interest using droplet digital PCR (ddPCR) at the Clinical Research Laboratory, Örebro University Hospital.

Differential expression was analysed in Strand NGS Software suite with one-way ANOVA for unequal variances (Welch) followed by Benjamini-Hochberg correction of multiple testing. All 68 samples from the initial, larger study [5] were included. Statistical significance was set at a corrected $\mathrm{p}$-value $<0.05$ and biological significance at a fold change $>2$.

Detailed information on the sampling procedure, RNA isolation, creation of small RNA libraries, NGS, ddPCR, bioinformatic and statistical analyses has been reported previously [5].

The characteristics of the participants divided by GWG are shown in Table 1. The NGS results showed 14 differentially expressed miRNAs, both biologically and statistically significant, between SGA neonates exposed or not to LGWG. MiR-379-3p, miR-519e-3p, miR-369-5p, miR-105-5p, miR-542-5p, miR-330-5p, miR-380-3p, miR-133a-3p, miR-335-3p, miR-4532, miR-3065-5p, miR-190b, and miR-526-5p were upregulated, and miR-3679-5p was downregulated in SGA neonates exposed to LGWG. The ddPCR analysis confirmed the differential expression of miR-379-3p, miR-519e-3p, and miR-105-5p, but not that of miR-380-3p. MiR-193b, which served as a negative control, did not show differential expression in neither NGS or ddPCR analyses.

The bioinformatic analysis revealed 44 upstream regulators for the differentially expressed miRNAs including proteins essential for the processing and regulation of
miRNAs (AGO2, DICER1, and DDX17), components of the mitogen-activated protein kinase (MAPK) signalling pathway (BRAF, HRAS, MAPK11, Smad2/3, and MEF2), and factors involved in the insulin-insulin-like growth factor (IGF) pathway (IGF1R, INSR, and insulin). Downstream effects of the differentially expressed miRNAs targeted the Canonical Pathway "Cancer Drug Resistance by Drug Efflux" and 122 categorical annotations related to diseases and functions. Of these annotations, 47 were involved in processes linked to cellular development (including cellular proliferation, cell growth and movement, cell death and survival, and cell cycle regulation), 24 were involved in biological processes associated with cancer, and eight were involved in inflammation.

The bioinformatic analyses also identified 888 predicted targets for the differentially expressed miRNAs. A list of the 40 most statistically significantly predicted targets is presented in Table 2 .

The birth of an SGA neonate originates in maternal, placental or foetal abnormalities. In this study, associations between miRNA expression in placental tissue and a potential, maternal cause for poor foetal growth, LGWG, were investigated. Among the 14 differentially expressed miRNAs found here between SGA neonates exposed or not to LGWG, miRNAs encoded by the chromosome 14 miRNA cluster (C14MC) [7] and the chromosome 19 miRNA cluster (C19MC) [8] were identified, namely miR-369-5p, miR-379$3 p$ and miR-380-3p; and miR-519-3p and miR-526b-5p, respectively. These clusters are predominantly expressed in embryonic and placental tissue, indicating placental specificity. Dysregulated expression of miRNAs in these

Table 1: Characteristics of participating women and their small-for-gestational age neonates divided by gestational weight gain group.

\begin{tabular}{|c|c|c|c|}
\hline & $\begin{array}{r}\text { Low gestational weight gain group } \\
(\mathrm{n}=13)\end{array}$ & $\begin{array}{r}\text { Normal gestational weight gain group } \\
\qquad(\mathrm{n}=9)\end{array}$ & p-Value \\
\hline \multicolumn{4}{|l|}{ Maternal characteristics } \\
\hline Age, years & $31.8(22.6-40.7)$ & $27.8(21.9-35.6)$ & 0.167 \\
\hline Nulliparity & $9(69 \%)$ & $8(89 \%)$ & 0.360 \\
\hline Early pregnancy weight, kg & $61(51-72)$ & $63(52-68)$ & 0.639 \\
\hline Height, cm & $167(158-174)$ & $166(158-178)$ & 0.843 \\
\hline Early pregnancy BMI, kg/m² & $22.3(18.7-24.9)$ & $22.6(20.7-24.4)$ & 0.695 \\
\hline GWG, kg & $10(6-10)$ & $13(12-15)$ & $<0.001$ \\
\hline \multicolumn{4}{|l|}{ Characteristics of the neonates } \\
\hline Gestational age, weeks & $40.6(37.7-41.9)$ & $39.7(38.6-41.7)$ & 0.096 \\
\hline Sex, numbers of females & $3(23.1 \%)$ & $5(55.6 \%)$ & 0.187 \\
\hline Birth weight, $\mathrm{g}$ & $2,840(2,155-3,160)$ & $2,620(2,290-3,060)$ & 0.100 \\
\hline Birth length, $\mathrm{cm}$ & $48(45-51)$ & $49(45-50)$ & 0.564 \\
\hline Head circumference, $\mathrm{cm}$ & $34.0(30.0-35.0)$ & $33.0(32.0-35.0)$ & 0.164 \\
\hline Birth weight z-score & $-2.2(-3.4$ to -2.0$)$ & $-2.4(-3.0$ to -2.1$)$ & 0.431 \\
\hline Birth length z-score & $-2.1(-3.5$ to -0.8$)$ & $-2.2(-3.1$ to -0.8$)$ & 0.948 \\
\hline Head circumference z-score & $-1.8(-3.4$ to -0.9$)$ & $-1.7(-2.4$ to -1.2$)$ & 0.918 \\
\hline
\end{tabular}

Data are medians (min-max) or numbers (\%). Low gestational weight gain was defined as $\leq 10 \mathrm{~kg}$. Normal gestational weight gain was defined as 11.5-16.0 kg. BMI, body mass index; GWG, gestational weight gain. 
Table 2: Predicted targets for the 14 microRNAs found to be differentially expressed in the placentas of small-for-gestational age neonates exposed or not to poor maternal gestational weight gain sorted by statistical significance.

\begin{tabular}{|c|c|c|}
\hline mRNA & Differentially expressed miRNAs found in the study predicted to influence the respective target & p-Value \\
\hline MEIOB & miR-379-3p, miR-380-3p, miR-3065-5p, miR-105-5p, miR-335-3p & $<0.001$ \\
\hline CT47A6 (includes others) & miR-3065-5p, miR-190b, miR-379-3p, miR-380-3p & $<0.001$ \\
\hline GTF3C6 & miR-379-3p, miR-335-3p, miR-380-3p, miR-526b-5p & $<0.001$ \\
\hline MRPL1 & miR-3065-5p, miR-526b-5p, miR-379-3p, miR-380-3p, miR-105-5p, miR-335-3p & $<0.001$ \\
\hline SLC28A1 & $\begin{array}{l}\text { miR-519e-3p, miR-526b-5p, miR-105-5p, miR-3065-5p, miR-3065-3p, miR-379-3p, miR-4532, } \\
\text { miR-330-5p, miR-380-3p }\end{array}$ & $<0.001$ \\
\hline TCTE3 & miR-190b, miR-519e-3p, miR-379-3p, miR-380-3p & $<0.001$ \\
\hline MORC1 & hsa-miR-526b-5p, miR-542-5p, miR-105-5p, miR-3065-5p, miR-379-3p, miR-380-3p, miR-335-3p & $<0.001$ \\
\hline RNASEH2B & miR-190b, miR-526b-5p, miR-105-5p, miR-379-3p, miR-330-5p, miR-380-3p, miR-335-3p & $<0.001$ \\
\hline RSPH6A & miR-519e-3p, miR-335-3p, miR-330-5p & $<0.001$ \\
\hline HLA-DQB1 ${ }^{\mathrm{a}}$ & miR-190b, miR-526b-5p, miR-379-3p, miR-330-5p, miR-380-3p, miR-335-3p & $<0.001$ \\
\hline $\mathrm{EZR}^{\mathrm{b}}$ & miR-190b, miR-519e-3p, miR-3065-5p, miR-379-3p, miR-380-3p, miR-335-3p & $<0.001$ \\
\hline $\mathrm{SRD} 5 \mathrm{~A} 2^{\mathrm{b}}$ & miR-190b, miR-526b-5p, miR-105-5p, miR-379-3p, miR-330-5p, miR-380-3p, miR-335-3p & $<0.001$ \\
\hline MYOM3 & $\begin{array}{l}\text { miR-190b, miR-519e-3p, miR-526b-5p, miR-105-5p, miR-3065-5p, miR-379-3p, miR-330-5p, } \\
\text { miR-335-3p }\end{array}$ & $<0.001$ \\
\hline SUCLG1 ${ }^{\mathrm{a}}$ & miR-3065-5p, miR-3065-3p, miR-379-3p, miR-380-3p, miR-105-5p, miR-335-3p & $<0.001$ \\
\hline ATP23 & miR-3065-5p, miR-190b, miR-526b-5p, miR-379-3p, miR-380-3p, miR-105-5p & $<0.001$ \\
\hline $\mathrm{NLRP} 2^{\mathrm{b}}$ & miR-379-3p, miR-380-3p, miR-190b, miR-335-3p & $<0.001$ \\
\hline ID $4^{\mathrm{a}}$ & $\begin{array}{l}\text { miR-190b, miR-526b-5p, miR-3065-5p, miR-369-5p, miR-379-3p, miR-330-5p, miR-380-3p, } \\
\text { miR-335-3p }\end{array}$ & $<0.001$ \\
\hline $\operatorname{LGALS}^{\mathrm{a}}$ & $\begin{array}{l}\text { miR-190b, miR-519e-3p, miR-105-5p, miR-369-5p, miR-3065-3p, miR-330-5p, miR-380-3p, } \\
\text { miR-335-3p }\end{array}$ & $<0.001$ \\
\hline CERNA1 & miR-3065-5p, miR-190b, miR-526b-5p, miR-3065-3p, miR-379-3p, miR-330-5p & $<0.001$ \\
\hline ENO4 & miR-190b, miR-526b-5p, miR-105-5p, miR-3065-5p, miR-3065-3p, miR-379-3p, miR-380-3p & $<0.001$ \\
\hline LOC400710 & miR-3065-5p, miR-3065-3p, miR-542-5p, miR-330-5p, miR-105-5p, miR-335-3p & $<0.001$ \\
\hline TMEM45A & miR-3065-5p, miR-369-5p, miR-379-3p, miR-380-3p, miR-105-5p, miR-335-3p & $<0.001$ \\
\hline SGMS2 ${ }^{b}$ & $\begin{array}{l}\text { miR-190b, miR-519e-3p, miR-105-5p, miR-3065-5p, miR-3065-3p, miR-379-3p, miR-4532, } \\
\text { miR-380-3p, miR-335-3p }\end{array}$ & 0.001 \\
\hline TFDP1 & $\begin{array}{l}\text { miR-519e-3p, miR-526b-5p, miR-105-5p, miR-3065-5p, miR-3065-3p, miR-379-3p, miR-380-3p, } \\
\text { miR-335-3p }\end{array}$ & 0.001 \\
\hline PLAUR $^{b}$ & miR-105-5p, miR-3065-5p, miR-379-3p, miR-330-5p, miR-380-3p, miR-335-3p & 0.001 \\
\hline PHLPP1 ${ }^{\text {b }}$ & miR-190b, miR-105-5p, miR-3065-5p, miR-379-3p, miR-330-5p, miR-380-3p, miR-335-3p & 0.001 \\
\hline ANP32AP1 & miR-190b, miR-335-3p, miR-526b-5p, miR-3065-3p & 0.001 \\
\hline CLIC2 & miR-519e-3p, miR-526b-5p, miR-105-5p, miR-3065-5p, miR-379-3p, miR-380-3p, miR-335-3p & 0.001 \\
\hline FAM120B & miR-526b-5p, miR-105-5p, miR-3065-5p, miR-3065-3p, miR-330-5p, miR-380-3p, miR-335-3p & 0.001 \\
\hline HCG22 & miR-3065-5p, miR-190b, miR-526b-5p, miR-542-5p, miR-330-5p, miR-380-3p, miR-335-3p & 0.001 \\
\hline $\mathrm{TNC}^{\mathrm{b}}$ & miR-526b-5p, miR-105-5p, miR-3065-5p, miR-379-3p, miR-330-5p, miR-380-3p, miR-335-3p & 0.001 \\
\hline CALCR & $\begin{array}{l}\text { miR-190b, miR-526b-5p, miR-105-5p, miR-3065-5p, miR-3065-3p, miR-379-3p, miR-330-5p, } \\
\text { miR-380-3p }\end{array}$ & 0.002 \\
\hline NRDE2 & miR-379-3p, miR-330-5p, miR-380-3p, miR-105-5p, miR-190b & 0.002 \\
\hline EXOC7 & miR-190b, miR-105-5p, miR-3065-3p, miR-379-3p, miR-330-5p, miR-380-3p, miR-335-3p & 0.002 \\
\hline C4orf19 & $\begin{array}{l}\text { miR-190b, miR-519e-3p, miR-526b-5p, miR-105-5p, miR-379-3p, miR-330-5p, miR-380-3p, } \\
\text { miR-335-3p }\end{array}$ & 0.002 \\
\hline AP3D1 & miR-3065-5p, miR-190b, miR-379-3p, miR-542-5p, miR-380-3p, miR-335-3p & 0.002 \\
\hline CCDC47 & $\begin{array}{l}\text { miR-526b-5p, miR-105-5p, miR-3065-5p, miR-3065-3p, miR-379-3p, miR-330-5p, miR-380-3p, } \\
\text { miR-335-3p }\end{array}$ & 0.002 \\
\hline ITPR2 & $\begin{array}{l}\text { miR-190b, miR-519e-3p, miR-526b-5p, miR-105-5p, miR-3065-5p, miR-379-3p, miR-330-5p, } \\
\text { miR-380-3p, miR-335-3p }\end{array}$ & 0.002 \\
\hline GK2 & miR-379-3p, miR-380-3p, miR-3065-5p, miR-335-3p & 0.002 \\
\hline ZNF202 & miR-190b, miR-519e-3p, miR-526b-5p, miR-3065-5p, miR-379-3p, miR-380-3p, miR-335-3p & 0.002 \\
\hline
\end{tabular}

Of 888 predicted targets, the 40 with the highest statistical significance are shown in the list together with the miRNAs predicted to influence the

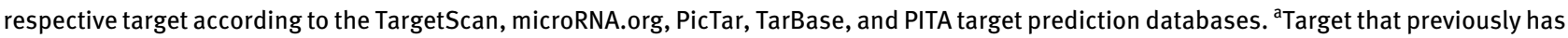
been associated with foetal growth or preeclampsia. ${ }^{\mathrm{b}}$ Target that previously has been associated with the insulin-IGF or MAPK signalling pathway. 
clusters has been associated previously with preeclampsia, foetal growth restriction (FGR), preterm birth, and tumour development $[3,8]$. Our findings show that expression of these miRNAs may not only differ by birth size and gestational age, but also by foetal exposure to normal or poor GWG in SGA neonates.

Both some of the upstream regulators and predicted targets (see Table 2) for the differentially expressed miRNAs found in this study were associated with the insulinIGF or MAPK pathways. These pathways are linked to each other, stimulate cellular proliferation and pre- and postnatal growth [9]. Insulin and IGF-1 are strongly regulated by nutritional intake linking food intake to growth. Dysregulation of IGF expression in placenta has previously been associated with restricted pre- and postnatal growth [1]. MiR-379-3p, which was upregulated in the placentas of SGA neonates exposed to LGWG in this study, has been shown to inhibit the expression of IGF-1 and concomitantly inhibit cell proliferation, invasion and migration [10].

The disease and function analysis of the differentially expressed miRNAs showed several annotations related to imperative cellular functions such as cellular proliferation, growth and movement, indicating that these basic functions may be differentially regulated in different subgroups of SGA neonates.

Limitations of this study include the small sample size, the restrictive entry criteria decreasing the possibility to generalize our findings to all SGA neonates, and the lack of verification of FGR by ultrasonography.

In conclusion, the expression of miRNAs in placenta from neonates born SGA exposed or not to LGWG is differential. Future studies are needed for the verification of our results in other populations and for detailed investigations on how miRNAs in the placenta influence foetal growth in relation to differences in exposure.

Acknowledgments: We thank all the women who participated and the midwives at the antenatal care units and the Department of Women's Health, Örebro University Hospital, involved in the project.
Research funding: This work was supported by the Research Committee and the ALF Funding of Region Örebro County. Author contributions: All authors have accepted responsibility for the entire content of this manuscript and approved its submission.

Competing interests: Authors state no conflict of interest. Informed consent: Informed consent was obtained from all individuals included in this study.

Ethical approval: The project was approved by the Regional Board of Ethics, Uppsala, Sweden (2010/189).

\section{References}

1. Saenger P, Czernichow P, Hughes I, Reiter EO. Small for gestational age: short stature and beyond. Endocr Rev 2007;28: 219-51.

2. Institute of Medicine (U.S.), Rasmussen KM, Yaktine AL, editors. Weight gain during pregnancy: reexamining the guidelines. Washington, DC: National Academies Press; 2009.

3. Catalanotto C, Cogoni C, Zardo G. MicroRNA in control of gene expression: an overview of nuclear functions. IJMS 2016;17:1712.

4. Thamotharan S, Chu A, Kempf K, Janzen C, Grogan T, Elashoff DA, et al. Differential microRNA expression in human placentas of term intra-uterine growth restriction that regulates target genes mediating angiogenesis and amino acid transport. PLoS One 2017;12:e0176493.

5. Östling H, Kruse R, Helenius G, Lodefalk M. Placental expression of microRNAs in infants born small for gestational age. Placenta 2019;81:46-53.

6. Niklasson A, Albertsson-Wikland K. Continuous growth reference from 24th week of gestation to 24 months by gender. BMC Pediatr 2008;29:8.

7. Wommack JC, Trzeciakowski JP, Miranda RC, Stowe RP, Ruiz RJ. Micro RNA clusters in maternal plasma are associated with preterm birth and infant outcomes. PLoS One 2018;13:e0199029.

8. Morales-Prieto DM, Ospina-Prieto S, Chaiwangyen W, Schoenleben M, Markert UR. Pregnancy-associated miRNA-clusters. J Reprod Immunol 2013;97:51-61.

9. Oldham S, Hafen E. Insulin/IGF and target of rapamycin signaling: a TOR de force in growth control. Trends Cell Biol 2003;13:79-85.

10. Li K, Wang Y, Zhang A, Liu B, Jia L. miR-379 inhibits cell proliferation, invasion, and migration of vascular smooth muscle cells by targeting insulin-like factor-1. Yonsei Med J 2017;58: 234-40. 